\title{
Analysis of the VROA signals of helical heptasilanes using an atomistic approach
}

\author{
Vincent Liégeois ${ }^{\mathrm{a}, *}$, Olivier Quinet ${ }^{\mathrm{a}}$, Benoît Champagne ${ }^{\mathrm{a}}$, \\ Jacques Haesler $^{\mathrm{b}}$, Gérard Zuber ${ }^{\mathrm{b}}$, Werner Hug ${ }^{\mathrm{b}}$ \\ ${ }^{a}$ Laboratoire de Chimie Théorique Appliquée, Facultés Universitaires, Notre-Dame de la Paix, \\ rue de Bruxelles, 61, B-5000 Namur, Belgium \\ ${ }^{\mathrm{b}}$ Département de Chimie, Université de Fribourg, ch. du Musée 9, CH-1700 Fribourg, Switzerland
}

\begin{abstract}
The VROA signatures of different helical conformers of heptasilane have been analyzed using an atomistic approach. Based on ab initio VROA quantities, the decomposition scheme and its representation under the form of group coupling matrices enable us to highlight the dominant role of the $\mathrm{Si}$ atoms for the modes studied. The sum of the contributions from $\mathrm{H}$ atoms, on the other hand, is generally negligible, often due to compensations, whereas the heteronuclear $\mathrm{Si}-\mathrm{H}$ contributions can be important. The dominating VROA signatures are mostly associated with collective wagging, rocking, and twisting motions.
\end{abstract}

Keywords: Raman optical activity; Group coupling matrix; Helical polymers

\section{Introduction}

First evidenced in the 70s [1], the vibrationnal Raman optical activity (VROA) spectroscopy has become a powerful technique to study chiral species such as fragrances [2,3], proteins [4-6], and natural products [7,8]. Recently, a theoretical investigation demonstrated that the VROA signatures are also specific to the helicity of synthetic polymers, and in particular polysilane chains [9]. Like vibrational circular dichroism (VCD) [10], its major advantage, as compared to electronic circular dichroism and optical rotatory dispersion, derives from the large number of vibrational signatures, each of them being a potential marker of the molecular structure [1113]. On the other hand, understanding this huge amount of structural information often requires interpretation tools associated with theoretical simulations of the spectra. In this respect, several computational schemes have been elaborated, implemented and validated on model compounds [3,7-9,14$23]$ in view of applications to specific problems. Moreover, a convenient approach has been proposed by one of us [24] to rationalize computed spectral intensities. It consists in the

\footnotetext{
* Corresponding author.
}

decomposition of the total VROA intensity of each vibrational mode into quasi-atomic and/or quasi-group contributions. The approach was recently implemented as a Matlab program called VOAView and applied to a number of organic molecules $[2,22,24]$. Here, this interpretation scheme is applied to discuss the VROA spectra of heptasilanes with different helicoidal conformations [9]. As evidenced for the IR and Raman spectra of conjugated quasi-linear systems [25-27], the vibrational intensities of oligomers are expected to highlight collective features.

The present paper is organized as follows: Section 2 summarizes the basic aspects of VROA intensities and their atomic decompositions. The computational aspects are detailed in Section 3. Section 4 describes the VOAView program and its functionality. Section 5 presents and discusses the results while conclusions are drawn in Section 6.

\section{Simulation and visualisation aspects}

For comparison with experiment, differential VROA scattering cross sections are used. In the harmonic approximation, the VROA backward differential scattering cross section per molecule, for an isotropic ensemble of molecules with a statistical distribution of their spatial orientation, for the $p$ th 
vibrational normal mode, and for the scattered circular polarization (SCP) scheme, reads [28,29]:

$-\Delta^{n} d \sigma(\pi)_{\mathrm{SCP}}=\frac{2 K_{\mathrm{p}}}{c} \frac{\hbar}{2 \Delta \omega_{\mathrm{p}}}\left[24 \beta_{\mathrm{G}_{\mathrm{p}}}^{2}+8 \beta_{\mathrm{A}_{\mathrm{p}}}^{2}\right] \mathrm{d} \Omega$

where $\omega_{0}=2 \pi \nu_{0}$ is the pulsation of the laser beam, $\omega_{\mathrm{p}}$ the pulsation of the scattered light and $\Delta \omega_{\mathrm{p}}$ the difference between the two previous pulsations.

For $\sigma$ in $\mathrm{m}^{2}$, the constant $K_{\mathrm{p}}$ is given by:

$K_{\mathrm{p}}=\frac{1}{90}\left(\frac{\mu_{0}}{4 \pi}\right)^{2} \omega_{\mathrm{p}}^{3} \omega_{0}$

The $\beta_{\mathrm{G}_{\mathrm{p}}}^{2}$ and $\beta_{\mathrm{A}_{\mathrm{p}}}^{2}$ VROA invariants, the anisotropic combinations of the derivatives with respect to the vibrational normal coordinate $Q_{\mathrm{p}}$ of the optical tensors $\left(\alpha_{\mu \nu}\right.$, the electric dipole-electric dipole polarizability; $A_{\lambda \kappa \nu}$, the electric dipole-electric quadrupole polarizability; $G_{\mu \nu}^{\prime}$, the electric dipole-magnetic dipole polarizability), have the form:

$\beta_{\mathrm{G}_{\mathrm{p}}}^{2}=\frac{1}{2} \sum_{\mu, \nu}\left[3\left(\frac{\partial \alpha_{\mu \nu}}{\partial Q_{\mathrm{p}}}\right)_{0}\left(\frac{\partial G_{\mu \nu}^{\prime}}{\partial Q_{\mathrm{p}}}\right)_{0}-\left(\frac{\partial \alpha_{\mu \mu}}{\partial Q_{\mathrm{p}}}\right)_{0}\left(\frac{\partial G_{\nu v}^{\prime}}{\partial \mathrm{Q}_{\mathrm{p}}}\right)_{0}\right]$

$\beta_{\mathrm{A}_{\mathrm{p}}}^{2}=\frac{\omega_{0}}{2} \sum_{\mu, \nu} \sum_{\lambda, \kappa}\left[\left(\frac{\partial \alpha_{\mu \nu}}{\partial Q_{\mathrm{p}}}\right)_{0}\left(\frac{\varepsilon_{\mu \lambda \kappa} \partial A_{\lambda \kappa \nu}}{\partial Q_{\mathrm{p}}}\right)_{0}\right]$

They can be expressed in terms of mono- $\left(J_{\alpha \alpha, \mathrm{p}}\right)$ and dinuclear $\left(J_{\alpha \beta, p}\right)$ invariant terms:

$$
\begin{aligned}
J_{\mathrm{p}} & =\sum_{\alpha, \beta}^{N} \sum_{i, j}^{3} L_{\alpha i, \mathrm{p}}^{x} V(J)_{\alpha i, \beta j} L_{\beta j, \mathrm{p}}^{x} \\
& =\sum_{\alpha, \beta} J_{\alpha \beta, \mathrm{p}}
\end{aligned}
$$

where $\underline{L}^{x}$ is the Cartesian displacement matrix of the normal modes normalized so that $\sum_{\alpha, i} m_{\alpha}\left(L_{\alpha i, \mathrm{p}}^{x}\right)^{2}=1$ and with $V_{\alpha i, \beta_{j}}$ for $J=\beta_{G}^{2}$ or $\beta_{\mathrm{A}}^{2}$ given by:

$$
\begin{aligned}
V\left(\beta_{\mathrm{G}}^{2}\right)_{\alpha i, \beta j}= & \frac{1}{2} \sum_{\mu, v}\left[3\left(\frac{\partial \alpha_{\mu \nu}}{\partial x_{i}^{\alpha}}\right)_{0}\left(\frac{\partial G_{\mu v}^{\prime}}{\partial x_{j}^{\beta}}\right)_{0}\right. \\
& \left.-\left(\frac{\partial \alpha_{\mu \mu}}{\partial x_{i}^{\alpha}}\right)_{0}\left(\frac{\partial G_{v v}^{\prime}}{\partial x_{j}^{\beta}}\right)_{0}\right] \\
V\left(\beta_{\mathrm{A}}^{2}\right)_{\alpha i, \beta j}= & \frac{\omega_{0}}{2} \sum_{u, v} \sum_{\lambda, \kappa}\left[\left(\frac{\partial \alpha_{\mu v}}{\partial x_{i}^{\alpha}}\right)_{0}\left(\frac{\varepsilon_{\mu \lambda \kappa} \partial A_{\lambda \kappa \nu}}{\partial x_{j}^{\beta}}\right)_{0}\right]
\end{aligned}
$$

In the above summations, the indices $\alpha$ and $\beta$ stand for atoms while $i$ and $j$ are used for Cartesian components $(x, y, z) . \varepsilon_{\mu \lambda \kappa}$ is the antisymmetric unit tensor of Levi-Civita.

The group coupling matrices (GCM) introduced in [24] provide a tool to represent the self-contributions of each atom $\left(J_{\alpha \alpha, \mathrm{p}}\right)$ to the VROA intensity as well as the contributions arising from coupling between atoms $\left(J_{\alpha \beta, \mathrm{p}}\right)$. Using straightforward summations, these definitions can be generalized to groups of atoms. For Raman, the GCM are symmetric, and for
ROA, in general non-symmetric matrices, with a dimension equal to the number of atoms or groups of atoms. The diagonal terms are mononuclear or intragroup while the off-diagonal terms are di-nuclear or intergroup, respectively. As only the symmetric part contributes to the total intensity of vibrational mode, they are best represented by upper triangular matrices, with off-diagonal elements equal to the sum of the two offdiagonal halves of the full matrix. Instead of numbers, the cases contain circles. The surface of the circles is chosen proportional to the $J_{\alpha \beta, \mathrm{p}}$ values whereas their color is related to their sign. The factor of proportionality is tunabled but fixed throughout this work for consistency of their analysis.

\section{Computational aspects}

The normal modes of vibration and their frequencies have been determined adopting the analytical coupled-perturbed Kohn-Sham [30] procedure and the 6-31G* [31] basis set. The hybrid B3LYP exchange-correlation functional has been selected owing to its recognized performances [32]. To account for missing electron correlation and anharmonicity effects, a multiplicative factor of 0.96 [32] is employed to scale the B3LYP vibrational frequencies.

A combination of analytical and numerical differentiation techniques has been employed to evaluate the first-order geometry derivatives of the optical tensors entering into the expression of the VROA invariants. These quantities have been calculated within the TDHF scheme [33,34] using the aug-ccpVDZ [35] basis set following recommendation of previous investigations $[9,20,21]$. In the case of the $\left(\partial \alpha_{\mu \nu} / \partial x\right)$ and $\left(\partial A_{\lambda \kappa v} /\right.$ $\partial x$ ), fully analytical procedures have been used [36,37]. The derivatives of the electric dipole-magnetic dipole polarizability $\left(G_{\mu \nu}^{\prime}\right)$ with respect to Cartesian coordinates are evaluated numerically. This is accomplished using a finite distortion procedure where the dynamic $G^{\prime}{ }_{\mu \nu}$ tensors are evaluated for distorted structures obtained by displacing each atom along the $x, y$ and $z$ axis. Four amplitudes of distortion have been used: \pm 0.005 and $\pm 0.010 \AA$, and a Romberg iteration has been employed to remove higher-order contaminations and therefore to achieve a sufficient accuracy on the numerical derivatives [38]. The $G^{\prime}$ tensors have been evaluated analytically at the TDHF level using the Gauge-Invariant Atomic Orbital (GIAO) approach $[39,40]$.

The calculations have been performed using both the GAMESS [41] and GAUSSIAN03 [42] quantum chemistry packages. The GAMESS program was used to get the analytical geometry derivatives of the $\left(\partial \alpha_{\mu \nu} / \partial x\right)$ and $\left(\partial A_{\lambda \kappa \nu} /\right.$ $\partial x$ ) optical tensors. GAUSSIAN03 was used to evaluate the TDHF optical activity tensors for distorted molecular geometries along the Cartesian axis as well as to determine the vibrational normal modes and frequencies. The typical $532 \mathrm{~nm}$ incident light wavelength has been adopted in all optical tensor calculations. A Maxwell-Boltzmann T-dependent factor has been added to Eq. (1) with $T=298.15 \mathrm{~K}$. In the spectra, each transition is represented by a Lorentzian function with a FWHM of $5 \mathrm{~cm}^{-1}$. 


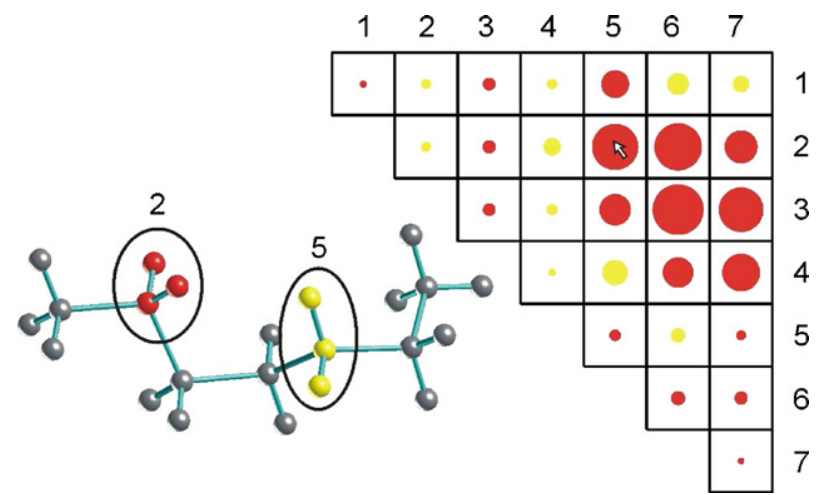

Fig. 1. Illustration of the user-friendly graphical interface used in VOAView. A simple mouse click on an element of the GCM highlights those atoms of the molecule giving rise to it. In this particular case, atoms colored in red belong to group 2 and those colored in yellow belong to group 5 .

\section{VOAView}

The Matlab code used in this paper to generate the atomic displacements and the pictures of the group coupling matrices is part of a graphical package called VOAView. Its main goal is to provide a tool to facilitate the interpretation of the vast amount of data available from the computation of Raman, ROA, IR and VCD.

VOAView uses its own input format. Different parsers are available to read outputs from the GAMESS, DALTON and GAUSSIAN programs. The main window allows the user to have a look at, and to rotate the calculated molecular structure. Atomic contribution patterns (ACP) and group coupling matrices (GCM) [24] are calculated internally. The groups for a GCM can be generated by a user friendly interface: they can be formed by a simple mouse click on the pertinent atoms. Conversely, a click on an element of a GCM highlights those atoms in a molecule which give rise to it. This is shown in Fig. 1.

\section{Results and discussion}

Fig. 2 displays the SCP spectra of the three helical conformers. The normal modes from 200 to $470 \mathrm{~cm}^{-1}$ present the most intense and distinct VROA bands and are the main focus of this analysis. Representations by spheres centered on nuclei, with surfaces proportional to the displacements, are displayed in Fig. 3. The GCMs for four choices of group distributions are shown in Figs. 4-7. In the first one, a group is made from a $\mathrm{SiH}_{2}$ (or $\mathrm{SiH}_{3}$ ) unit so that the whole system is divided into seven groups. In the second one, there are only two groups, one with all $\mathrm{Si}$ atoms, the other with all $\mathrm{H}$ atoms. In the last two GCM representations, the $\mathrm{Si}$ and $\mathrm{H}$ atoms are treated separately. In the case of the Si atoms, there are seven groups, each containing one atom, while in the case of the $\mathrm{H}$ atoms, there are also seven groups but with each containing the two or three $\mathrm{H}$ atoms attached to an individual $\mathrm{Si}$ atom. These representations are provided for a selection of modes, associated with the intense couplet around $450 \mathrm{~cm}^{-1}$ (see below), the other ones, for space consideration, are given as supplementary materials available on the site available on Journal Spectroscopy website see Appendix A.
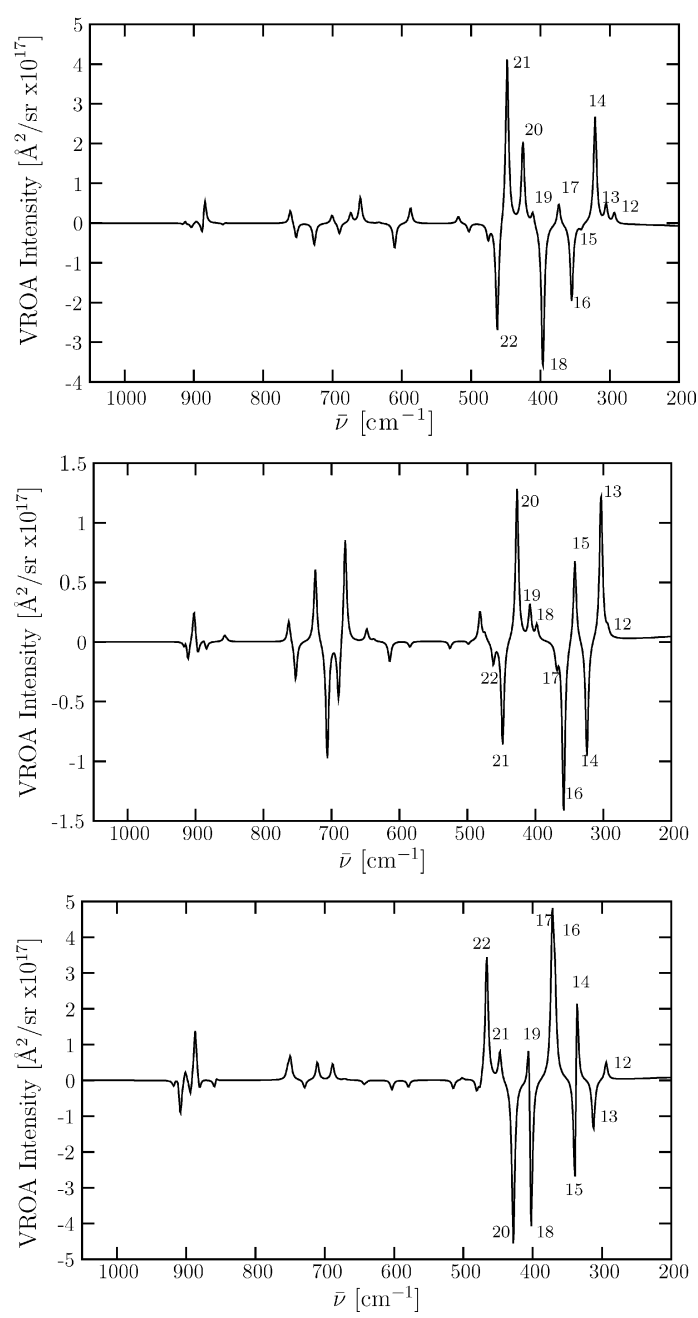

Fig. 2. Simulated VROA backward spectra of TGTG (top), TGTG' (center), and GGGG (bottom) heptasilanes. The vibrational normal coordinates have been evaluated at the B3LYP/6-31G* level whereas the polarizabilities and their geometry derivatives at the TDHF/aug-cc-pVDZ level. Each transition is represented by a Lorentzian function with FWHM of $5 \mathrm{~cm}^{-1}$. The optical wavelength is $532 \mathrm{~nm}$ and a multiplicative factor of 0.96 is used to scale the vibrational frequencies.

The different frequency ranges of the VROA spectra between 200 and $470 \mathrm{~cm}^{-1}$ have been successively studied. First, an intense couplet in the vicinity of $450 \mathrm{~cm}^{-1}$, due to collective motions, has been considered. It corresponds to the normal modes 21 and 22 for the TGTG conformer, 20 and 21 for the TGTG' conformer, 20 and 22 for the GGGG conformer. For all conformers, these modes are characterized by out-ofphase wagging motions of the $\mathrm{H}$ atoms together with $\mathrm{Si}-\mathrm{Si}$ stretching motions. Going towards lower vibrational frequencies, the modes presenting large VROA intensities have rocking and twisting characters. Indeed, for the TGTG conformer, mode 18 is a mixing of rocking and twisting motions of the $\mathrm{H}$ atoms together with a slight $\mathrm{Si}-\mathrm{Si}$ stretching. Mode 16 is mostly rocking motions of the $\mathrm{H}$ atoms combined with twisting motions of the central $\mathrm{SiH}_{2}$ unit and $\mathrm{Si}-\mathrm{Si}$ stretching. Mode 14 corresponds to rocking displacements in one halve of the molecule. For TGTG', mode 16 corresponds to rocking motions with twisting of the central $\mathrm{SiH}_{2}$ unit. Mode 


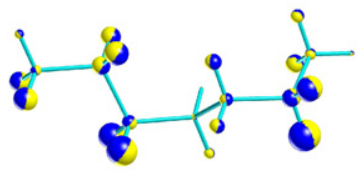

Mode 22

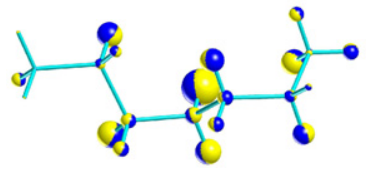

TGTG'

Mode 20

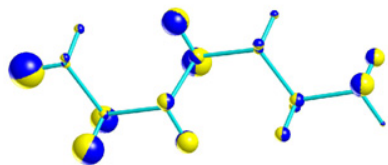

Mode 21

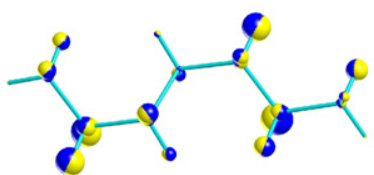

GGGG

Mode 20

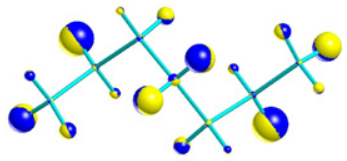

Mode 22

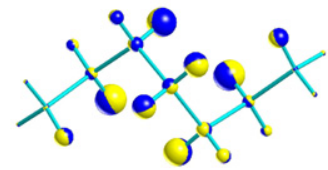

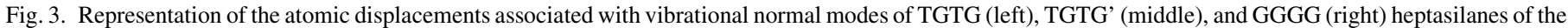

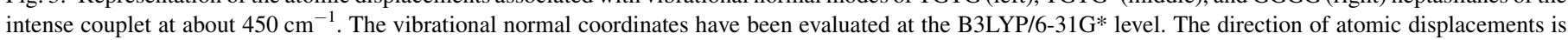
perpendicular to the junction plane between the two hemispheres of distinct color while their amplitudes are proportional to the sphere surface.

15 is a combination of rocking motions with $\mathrm{Si}-\mathrm{Si}$ stretching motions. Modes 13 and 14 are mostly rocking motions. Then, for the GGGG conformer, mode 18 involves rocking motions of the three central $\mathrm{SiH}_{2}$ units. Modes 16 and 17 are also rocking motions but they spread over the whole molecule. Mode 15 is the equivalent of mode 18 in the TGTG conformer. Mode 14 consists of rocking motions. In mode 23 and higher frequency modes, the units also exhibit wagging and rocking
TGTG

Mode 21

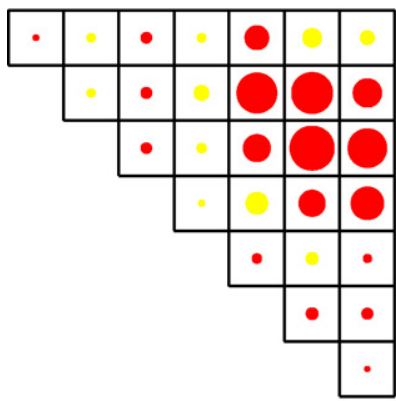

Mode 22

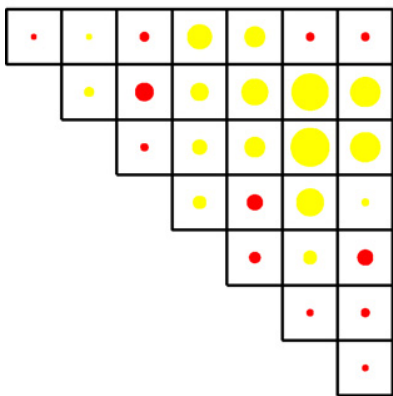

TGTG'

Mode 20

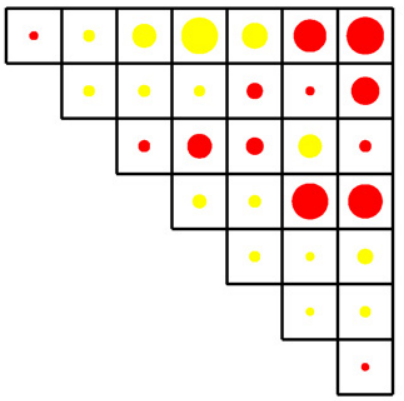

Mode 21

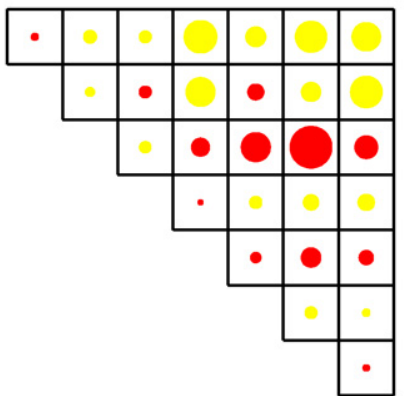

GGGG

Mode 20

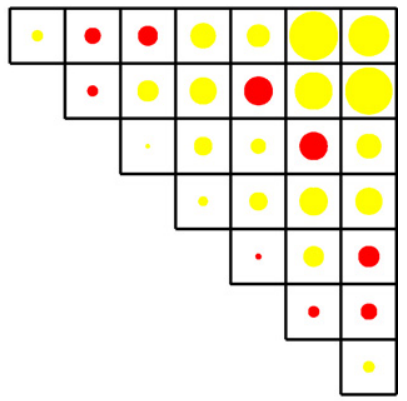

Mode 22

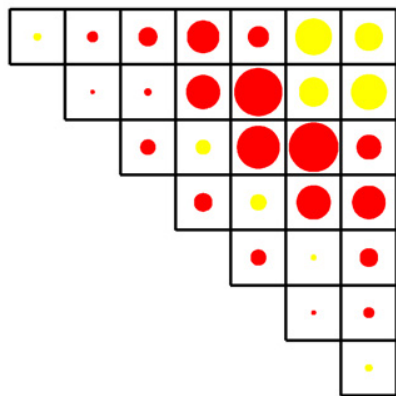

Fig. 4. Representation of the GCM matrix elements of the intense couplet at about $450 \mathrm{~cm}^{-1}$ for the seven $\mathrm{SiH}_{2}\left(\mathrm{SiH}_{3}\right)$ groups. Indices of the matrices are associated to the corresponding units of TGTG (left), TGTG' (middle), and GGGG (right) heptasilane chains. 
TGTG

Mode 21

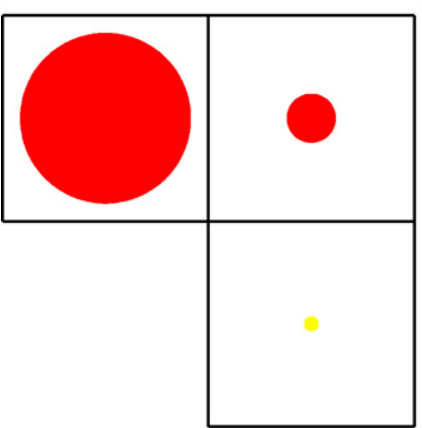

Mode 22

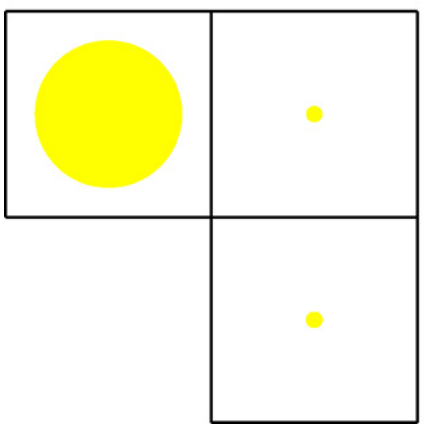

TGTG'

Mode 20

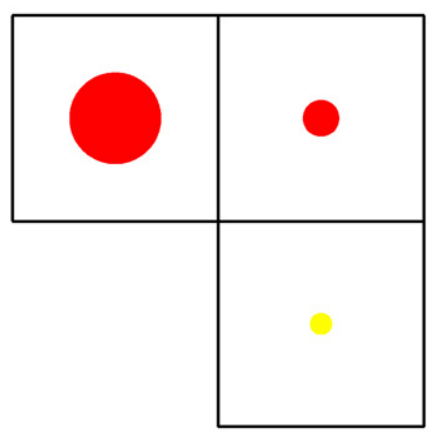

Mode 21

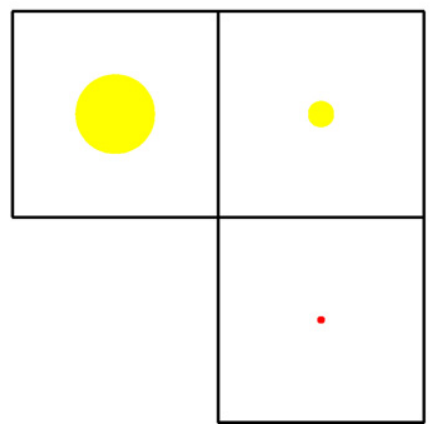

GGGG

Mode 20

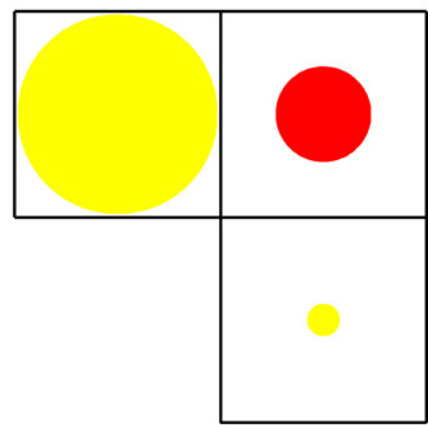

Mode 22

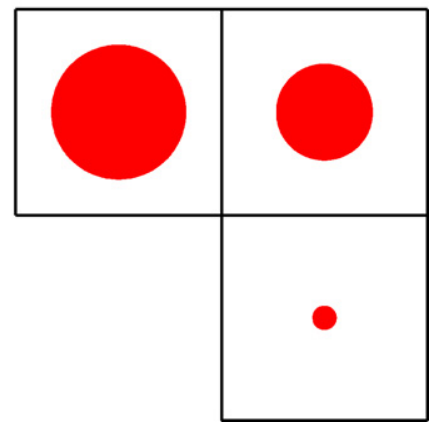

Fig. 5. Representation of the GCM matrix elements of the intense couplet at about $450 \mathrm{~cm}^{-1}$ for the two sets of atoms. The first indice is associated to the Si atoms while the second to the H atoms of TGTG (left), TGTG' (middle), and GGGG (right) heptasilane chains.

TGTG

Mode 21

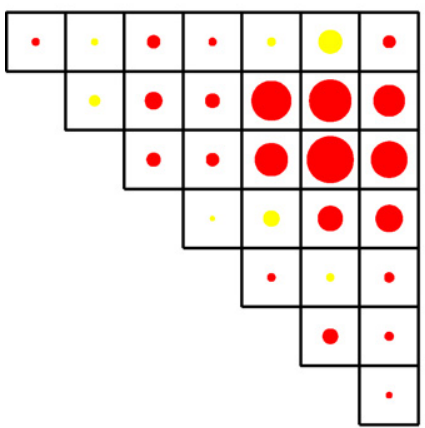

Mode 22

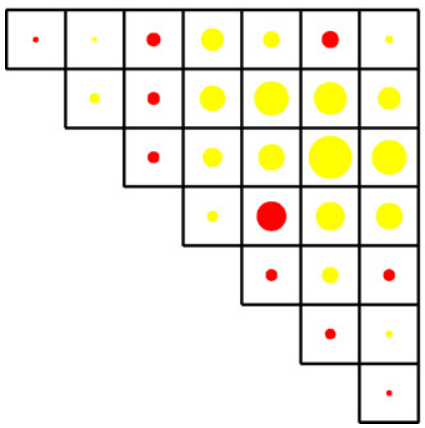

TGTG'

Mode 20

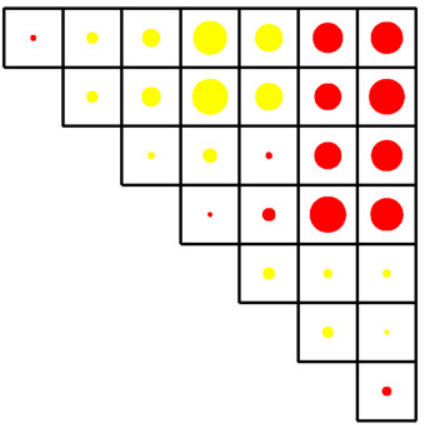

Mode 21

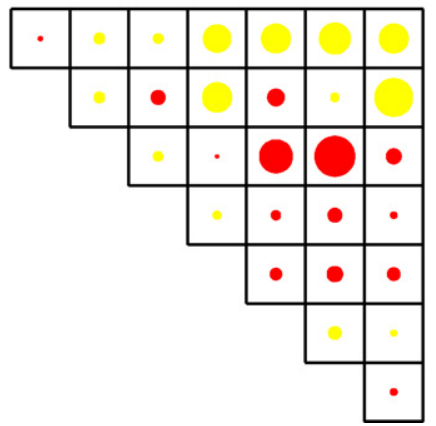

GGGG

Mode 20

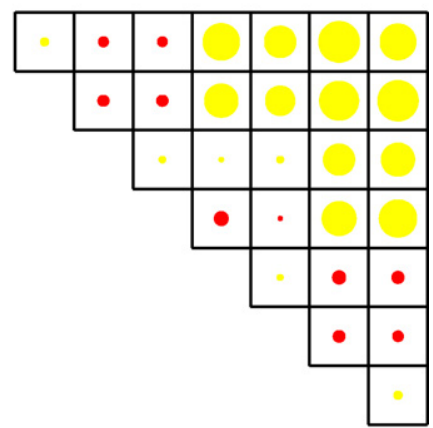

Mode 22

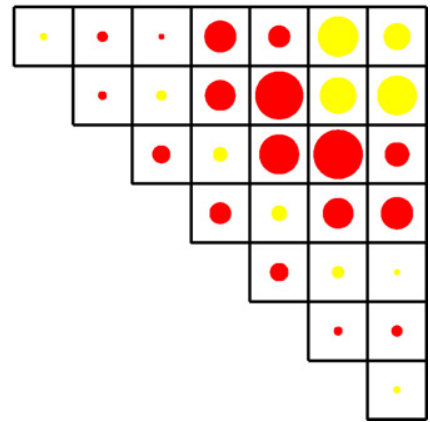

Fig. 6. Representation of the GCM matrix elements of the intense couplet at about $450 \mathrm{~cm}^{-1}$ for the seven groups of Si atoms. Indices of the matrices are associated to the corresponding units of TGTG (left), TGTG' (middle), and GGGG (right) heptasilane chains. 
TGTG

Mode 21

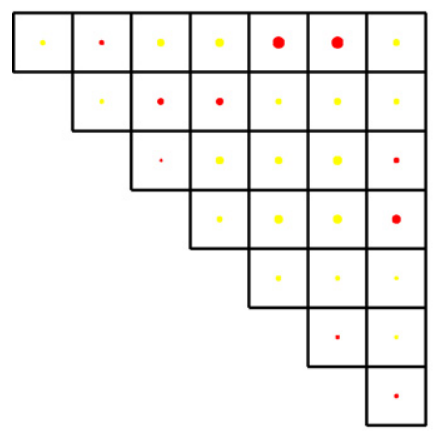

Mode 22

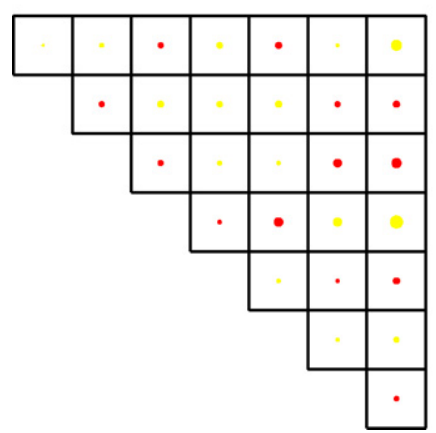

TGTG'

Mode 20

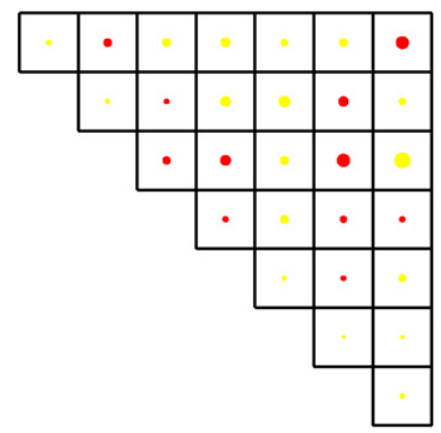

Mode 21

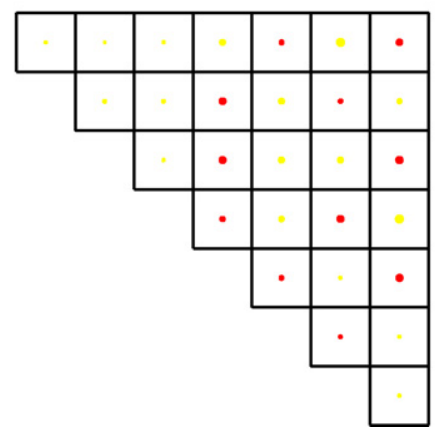

GGGG

Mode 20

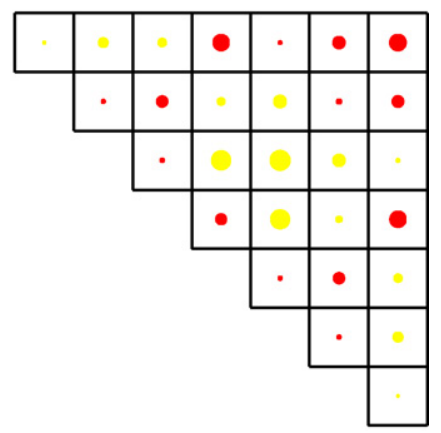

Mode 22

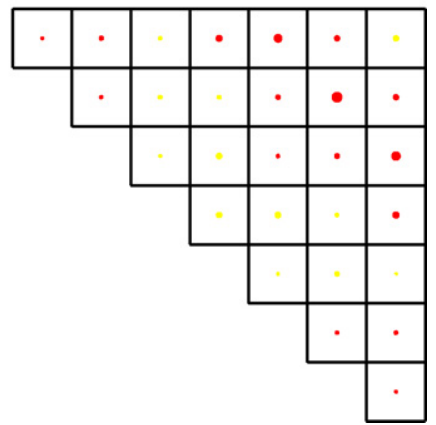

Fig. 7. Representation of the GCM matrix elements of the intense couplet at about $450 \mathrm{~cm}^{-1}$ for the seven groups of $\mathrm{H}$ atoms. Indices of the matrices are associated to the corresponding units of TGTG (left), TGTG' (middle), and GGGG (right) heptasilane chains.

motions but, as explained further, they are associated with smaller VROA intensities.

For the $450 \mathrm{~cm}^{-1}$ couplet, it is evident from Fig. 5 that the combination of Si motions, together with the gradients at the $\mathrm{Si}$ nuclei, make the largest contributions to the VROA intensities. The next contribution, in magnitude, is heteronuclear and originates from the coupling between the $\mathrm{H}$ and $\mathrm{Si}$ motions. The homonuclear $\mathrm{H}$ contributions, in contrast, are negligible. The dominance of the $\mathrm{Si}$ contribution is also visible from the comparison of Fig. 4 with Fig. 6, where one must take into account that Fig. 4 is the sum of Figs. 6 and 7 and all the crossterms between $\mathrm{Si}$ and $\mathrm{H}$ atoms.
For mode 18 in the TGTG conformer, the Si homonuclear interactions (Fig. 6 of supplementary materials) leading to the important negative VROA intensity are associated with pairs of non-bonded $\mathrm{Si}$ atoms: $\mathrm{Si}_{1}-\mathrm{Si}_{4}, \mathrm{Si}_{1}-\mathrm{Si}_{5}, \mathrm{Si}_{2}-\mathrm{Si}_{4}, \mathrm{Si}_{2}-\mathrm{Si}_{5}$ and their symmetrical analogs $\mathrm{Si}_{7}-\mathrm{Si}_{4}, \mathrm{Si}_{7}-\mathrm{Si}_{3}, \mathrm{Si}_{6}-\mathrm{Si}_{4}, \mathrm{Si}_{6}-\mathrm{Si}_{3}$, which shows that VROA intensity generation can also have a longrange character. Nevertheless, the contributions of the $\mathrm{SiH}_{2}$ groups are different from the Si ones since the symmetry in the matrix is broken. Indeed, the heteronuclear $\mathrm{Si}-\mathrm{H}$ contributions present another pattern such that the important negative values associated with the $\mathrm{SiH}_{2} / \mathrm{SiH}_{3}$ units are finally associated with unit 2 interacting with units 5,6 and 7; with unit 3 interacting

Table 1

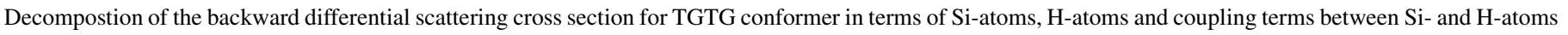

\begin{tabular}{llcrr}
\hline Mode & $-\Delta^{n} d \sigma(\pi)_{\mathrm{Si}}\left(10^{-17} \AA^{2} / \mathrm{sr}\right)$ & $-\Delta^{n} d \sigma(\pi)_{\mathrm{H}}\left(10^{-17} \AA^{2} / \mathrm{sr}\right)$ & $-\Delta^{n} d \sigma(\pi)_{\mathrm{Si}-\mathrm{H}}\left(10^{-17} \AA^{2} / \mathrm{sr}\right)$ & $-\Delta^{n} d \sigma(\pi)_{\mathrm{SCP}}\left(10^{-17} \AA^{2} / \mathrm{sr}\right)$ \\
\hline 12 & 0.0363 & 0.2596 & -0.0349 & 0.2610 \\
13 & 0.0382 & 0.2052 & 0.2133 & 0.4567 \\
14 & 1.3186 & 0.3161 & 1.0772 & 2.7119 \\
15 & 0.9609 & -0.1152 & -0.9706 & -0.1249 \\
16 & -1.9634 & 0.0942 & -0.0874 & -1.9566 \\
17 & -0.2379 & -0.1486 & 0.9559 & 0.5694 \\
18 & -2.6866 & -0.0303 & -1.0057 & -3.7226 \\
19 & -0.2771 & -0.0364 & 0.6278 & 0.3143 \\
20 & 2.7186 & 0.0402 & -0.7009 & 2.0579 \\
21 & 3.9196 & -0.0193 & 0.2970 & 4.1973 \\
22 & -2.7867 & -0.0247 & -0.0233 & -2.8347 \\
\hline
\end{tabular}


Table 2

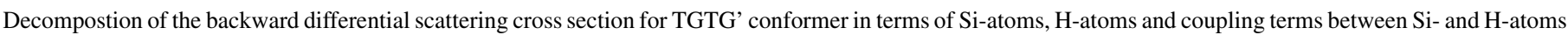

\begin{tabular}{|c|c|c|c|c|}
\hline Mode & $-\Delta^{n} d \sigma(\pi)_{\mathrm{Si}}\left(10^{-17} \AA^{2} / \mathrm{sr}\right)$ & $-\Delta^{n} d \sigma(\pi)_{\mathrm{H}}\left(10^{-17} \AA^{2} / \mathrm{sr}\right)$ & $-\Delta^{n} d \sigma(\pi)_{\mathrm{Si}-\mathrm{H}}\left(10^{-17} \AA^{2} / \mathrm{sr}\right)$ & $-\Delta^{n} d \sigma(\pi)_{\mathrm{SCP}}\left(10^{-17} \AA^{2} / \mathrm{sr}\right)$ \\
\hline 12 & 0.0304 & 0.0386 & -0.0038 & 0.0652 \\
\hline 13 & 0.2879 & 0.4105 & 0.5503 & 1.2487 \\
\hline 14 & -0.2600 & -0.2915 & -0.4489 & -1.0004 \\
\hline 15 & 1.0211 & 0.0207 & -0.3328 & 0.7090 \\
\hline 16 & -0.9012 & -0.1044 & -0.4604 & -1.4660 \\
\hline 17 & 0.2706 & -0.0241 & -0.4251 & -0.1786 \\
\hline 18 & -1.5038 & -0.0827 & 1.7093 & 0.1228 \\
\hline 19 & 0.4507 & -0.1231 & -0.0389 & 0.2887 \\
\hline 20 & 1.1695 & -0.0531 & 0.1656 & 1.2820 \\
\hline 21 & -0.8172 & 0.0028 & -0.0744 & -0.8888 \\
\hline 22 & 0.3237 & -0.0327 & -0.4758 & -0.1848 \\
\hline
\end{tabular}

Table 3

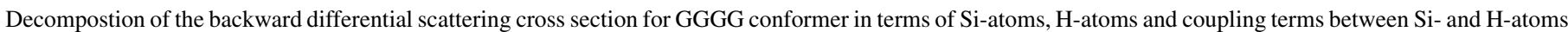

\begin{tabular}{|c|c|c|c|c|}
\hline Mode & $-\Delta^{n} d \sigma(\pi)_{\mathrm{Si}}\left(10^{-17} \AA^{2} / \mathrm{sr}\right)$ & $-\Delta^{n} d \sigma(\pi)_{\mathrm{H}}\left(10^{-17} \AA^{2} / \mathrm{sr}\right)$ & $-\Delta^{n} d \sigma(\pi)_{\mathrm{Si}-\mathrm{H}}\left(10^{-17} \AA^{2} / \mathrm{sr}\right)$ & $-\Delta^{n} d \sigma(\pi)_{\mathrm{SCP}}\left(10^{-17} \AA^{2} / \mathrm{sr}\right)$ \\
\hline 12 & 0.1749 & 0.0925 & 0.2228 & 0.4902 \\
\hline 13 & -1.4353 & 0.1612 & -0.1471 & -1.4212 \\
\hline 14 & 2.0506 & 0.2728 & 2.2447 & 4.5681 \\
\hline 15 & -5.2443 & 0.0431 & 0.1548 & -5.0464 \\
\hline 16 & 2.8591 & 0.0224 & -0.7046 & 2.1769 \\
\hline 17 & 3.2760 & -0.0695 & 1.0006 & 4.2071 \\
\hline 18 & -2.4309 & -0.5389 & -2.2341 & -5.2039 \\
\hline 19 & 4.1429 & 0.1880 & -1.6299 & 2.7010 \\
\hline 20 & -5.7429 & -0.1286 & 1.2685 & -4.6030 \\
\hline 21 & 2.0901 & -0.1126 & -1.1573 & 0.8202 \\
\hline 22 & 2.2981 & 0.0591 & 1.1620 & 3.5192 \\
\hline
\end{tabular}

with units 5 and 7; and finally with unit 4 interacting with units 5, 6, and 7. This effect is due to the heteronuclear $\mathrm{Si}-\mathrm{H}$ contributions, which are, however, about three times smaller than the homonuclear $\mathrm{Si}$ contributions. For the GGGG conformer, more than for the other conformers, Figs. 4 and 6 of supplementary materials present a somewhat different picture, since in this case the individual homonuclear $\mathrm{H}$ terms are almost as large as the homonuclear Si contributions. Most homonuclear Si contributions, however, have an identical sign and so add up to large VROA, whereas for the $\mathrm{H}$ atoms, the signs differ and contributions therefore tend to cancel. There is, moreover, an interesting sign alternation with the distance for the $\mathrm{H}$ contributions. This pattern is found for modes $12,13,15$, 16 , and 17 of the GGGG conformer as well as for modes 12, 13 of the TGTG conformer. The TGTG' conformer does not present similar GCM characteristics.

Tables 1-3 provide a numerical comparison of the contributions which the $\mathrm{Si}$-atoms, the H-atoms, and the coupling terms between $\mathrm{Si}$ - and $\mathrm{H}$-atoms make to the SCP scattering cross-sections in the modes with a collective character in the different conformers.

\section{Conclusions}

The VROA signatures of different helical conformers of heptasilane have been analyzed using the atomistic approach proposed in Ref. [24]. Based on recent calculated VROA intensities [9], the GCM decomposition scheme, together with a graphical representation of the matrices, enable us to highlight the dominant role which the Si atoms have for the VROA generation for the modes we consider, as well as the expected collective character of these vibrations. On the other hand, the contributions from the $\mathrm{H}$ atoms are generally negligible whereas the heteronuclear $\mathrm{Si}-\mathrm{H}$ contributions can be of importance, although they do not exhibit a similarly regular pattern as the $\mathrm{Si}$ contributions do. The dominating VROA signatures are mostly associated with wagging, rocking, and twisting motions. Future investigations will encompass the comparison between oligosilanes and alcanes as well as the study of isotopic substitutions. In addition, they will bridge the gap between model helical systems and real systems where substituents induce preferential handedness of the helices. Among these compounds, polypropylene chains with bulky substituents at their extremities adopt a helicity determined by the configuration of the terminal carbon atom.

\section{Acknowledgments}

This work is the result of a scientific collaboration within the D26 COST Action, financially supported by the European Science Foundation. V.L., O.Q., and B.C. thank the FNRS for their Research Fellow, Postdoctoral Researcher, and Research Director positions, respectively. The development of the VOAView program was supported by Swiss National Science Foundation grants 200020-103750 and 2000-066679, and the 
Swiss BF C03.0058 grant providing support for collaboration within the COST framework. The calculations have been performed on the Interuniversity Scientific Computation Facility (ISCF), installed at the Facultés Universitaires Notre-Dame de la Paix (Namur, Belgium), for which the authors gratefully acknowledge the financial support of the FNRS-FRFC and the Loterie Nationale for the Grant No. 2.4578.02, and of the FUNDP.

\section{Appendix A. Supplementary data}

Supplementary data associated with this article can be found, in the online version, at doi:10.1016/j.vibspec.2006.05.026.

\section{References}

[1] L.D. Barron, M.P. Bogaard, A.D. Buckingham, J. Am. Chem. Soc. 95 (1973) 603.

[2] G. Zuber, W. Hug, Helv. Chim. Acta 87 (2004) 2208.

[3] G. Zuber, M.-R. Goldsmith, D.N. Beratan, P. Wipf, ChemPhysChem 6 (2005) 595.

[4] I.H. McColl, E.W. Blanch, A.C. Gill, A.G.O. Rhie, M.A. Ritchie, L. Hecht, K. Nielsen, L.D. Barron, J. Am. Chem. Soc. 125 (2003) 10019.

[5] E.W. Blanch, A.C. Gill, A.G.O. Rhie, J. Hope, L. Hecht, K. Nielsen, L.D. Barron, J. Mol. Biol. 343 (2004) 467.

[6] I.H. McColl, E.W. Blanch, L. Hecht, L.D. Barron, J. Am. Chem. Soc. 126 (2004) 8181.

[7] K. Ruud, T. Helgaker, P. Bour, J. Phys. Chem. A 106 (2002) 7448.

[8] M. Pecul, A. Rizzo, J. Leszczynski, J. Phys. Chem. A 106 (2002) 11008.

[9] V. Liégeois, O. Quinet, B. Champagne, J. Chem. Phys. 122 (2005) 214304.

[10] P.J. Stephens, Ann. Rev. Phys. Chem. 36 (1985) 213.

[11] L.A. Nafie, Ann. Rev. Phys. Chem. 48 (1997) 357.

[12] L.D. Barron, L. Hecht, I.M. McColl, E.W. Blanch, Mol. Phys. 102 (2004) 731.

[13] W. Hug, Raman Optical Activity Spectroscopy, in: J.M. Chalmers, P.R. Griffiths (Eds.), Handbook of Vibrational Spectroscopy, vol. 1, John Wiley and Sons, Chichester, 2002.
[14] P.L. Polavarapu, J. Phys. Chem. 94 (1990) 8106.

[15] P.L. Polavarapu, L. Hecht, L.D. Barron, J. Phys. Chem. 97 (1993) 1793.

[16] T. Helgaker, K. Ruud, B.L. Bak, P. Jørgensen, J. Olsen, Faraday Discuss. 99 (1994) 165.

[17] P. Bour, J. Comput. Chem. 22 (2001) 426.

[18] K.J. Jalkanen, R.M. Nieminen, M. Knapp-Mohammady, S. Suhai, Int. J. Quant. Chem. 92 (2003) 239.

[19] M. Pecul, A. Rizzo, Mol. Phys. 101 (2003) 2073.

[20] G. Zuber, W. Hug, J. Phys. Chem. A 108 (2004) 2108.

[21] M. Reiher, V. Liégeois, K. Ruud, J. Phys. Chem. A 109 (2005) 7567.

[22] W. Hug, J. Haesler, Int. J. Quant. Chem. 104 (2005) 695.

[23] M. Pecul, K. Ruud, Int. J. Quant. Chem. 104 (2005) 816.

[24] W. Hug, Chem. Phys. 264 (2001) 53.

[25] G. Zerbi, M. Del Zoppo, J. Chem. Soc. Faraday Trans. 88 (1835).

[26] C.X. Cui, M. Kertesz, Macromolecules 25 (1993) 1103.

[27] B. Kirtman, B. Champagne, J. André, J. Chem. Phys. 104 (1996) 4125.

[28] W. Hug, in: J.M. Chalmers, P.R. Griffiths (Eds.), Handbook of Vibrational Spectroscopy., vol. 1, John Wiley and Sons, Chichester, 2002.

[29] L.D. Barron, Molecular Light Scattering and Optical Activity, Cambridge University Press, Cambridge, 2004.

[30] A. Komornicki, G. Fitzgerald, J. Chem. Phys. 98 (1993) 1398.

[31] W.J. Hehre, R. Ditchfield, J.A. Pople, J. Chem. Phys. 56 (1972) 2257.

[32] A.P. Scott, L. Radom, J. Phys. Chem. 100 (1996) 16502.

[33] H. Sekino, R.J. Bartlett, J. Chem. Phys. 85 (1986) 976.

[34] S.P. Karna, M. Dupuis, J. Comput. Chem. 12 (1991) 487.

[35] T.H. Dunning, J. Chem. Phys. 90 (1989) 1007.

[36] O. Quinet, B. Champagne, J. Chem. Phys. 115 (2001) 6293.

[37] O. Quinet, V. Liégeois, B. Champagne, J. Chem. Theory Comput. 1 (2005) 444.

[38] P.J. Davis, P. Rabinowitz, Numerical Integration, Blaisdell Publishing Company, London, 1967.

[39] R. Ditchfield, J. Am. Chem. Soc. 93 (1971) 5287.

[40] P.J. Stephens, F.J. Devlin, J.R. Cheeseman, M.J. Frisch, J. Phys. Chem. A 105 (2001) 5356.

[41] M.W. Schmidt, K.K. Baldridge, J.A. Boatz, S.T. Elbert, M.S. Gordon, J.H. Jensen, S. Koseki, N. Matsunaga, K.A. Nguyen, S.J. Su, T.L. Windus, M. Dupuis, J.A. Montgomery, J. Comp. Chem. 14 (1993) 1347.

[42] M.J. Frisch, et al., Gaussian 03, Revision B. 04, Gaussian, Inc., Wallingford, CT, 2004. 